\title{
Interactive comment on "Direct links between hygroscopicity and mixing state of ambient aerosols: Estimating particle hygroscopicity from their single particle mass spectra" by Xinning Wang et al.
}

\section{Anonymous Referee \#1}

Received and published: 17 January 2020

General comments: This paper introduces a method by connecting an aerosol timeof-flight mass spectrometer (ATOFMS) to the downstream of a hygroscopic tandem differential mobility analyzer (HTDMA), in order to simultaneously compare the hygroscopicity and composition of submicron aerosol. The author linked the particle types to the hygroscopicity, and showed the diversity distribution of hygroscopicity. Furthermore, they estimated ambient particle hygroscopicity from their mass spectra. The topic of the paper is well suited for ACP. The data are generally sound, whereas the results and discussions of the study are lack of persuasion. More effort needs to be 
put into the presentation of the results. I have some points where more information is needed or where I disagree.

(1) The authors showed that the temporal variations of the estimated particle hygroscopicity were consistent with the back-trajectory analysis and atmospheric visibility observations. It is hard to believe that hygroscopicity could be simply explained by the back-trajectory analysis, or the observed hygroscopicity could be a major reason for the visibility. A closer look at the discussion revealed that such conclusions were not precisely summarized.

(2) The results indicate that particles with stronger hygroscopicities were more likely to have higher effective densities. Is it suitable for all the observed particle types? Could it be theoretically supported? This is hard to believe. For instance, aging of Dust particles lead to nitrate coatings, which would lower the effective density, but increase the hygroscopicity.

Specific comments:

1. Abstract: "These hygroscopicity estimation results with single particle mass spectra analysis can provide critical information on particulate water content, particle source apportionment, and aging processes." I wonder how hygroscopicity can provide critical information on the source of particles. The authors did not discuss this in the text but only took sea salt particles as an example in Figure 5.

2. Introduction: "Herich et al. have firstly applied the HTDMA-ATOFMS system to investigate particle composition as a function of hygroscopicity." Some results related to such measurements are missing here.

3. Experimental section: what is the accuracy of hygroscopicity determination by the HTDMA? What is the uncertainty in GF prediction?

4. Line 148: "The DMAs were kept to select the desired diameters before significant number of particle were chemical analyzed by ATOFMS." What does a significant num- 
ber refer to?

5. Line 171: "This problem was relieved by taking the 0.5 power (square root) of peaks intensities." Maybe more information is required to validate such treatments.

6. Line 239: "About $20 \%$ of analyzed particles are classified as Amines-rich". Is there any explanation for such a high fraction of Amine particles?

Interactive

comment

7. Lines 251-252: “. . CNO peaks, which are present in biomass particles, were absent very weak, suggesting that biomass burning is not their source." Do you mean CN/CNO peaks are necessarily served as markers for biomass burning?

8. Line 256: ". . characterizations, it is possible that the ammonium/OC particles might be from coal burning sources". There are already some paper published reporting the single particle mass spectra of coal burning particles in China. The author should directly refer to these papers, rather than (Healy et al., 2010). Still, such an assignment might not be appropriate, since it is more likely produced from secondary processes, associated with high abundance of ammonium sulfate.

9. Line 260: the authors further concluded that "These particles were not likely to be deeply aged particles, because their hygroscopicity was only moderate." It is a little bit confusing, since the aging should be deduced from the mass spectra, not the hygroscopicity. Hygroscopicity could be linked to the chemical compositions, but not particle age.

10. Line 398: “. . higher hygroscopicity could play more important role. .." higher correlation cannot infer the higher contribution of aerosol particles with higher hygroscopicity to visibility decrease. Is it possible to estimate the relative contribution based on the combined measurements in this study?

Minor:

References such as "Zelenyuk et al.(Zelenyuk et al., 2008)" is incorrectly formatted. 


\section{Line 98: "HTMDA-ATOFMS"}

Line 183: "value from 0.1 to 1.7 " is it $0.9-1.7$ ?

ACPD

Line 314: "can be measured on by HTDMA-ATOMFS system"

Interactive comment on Atmos. Chem. Phys. Discuss., https://doi.org/10.5194/acp-2019-1018,

Interactive 2020. 